\title{
Determination of Plasma Tissue Factor Antigen and Tissue Factor-Bearing Microparticles Activity in Healthy Subjects
}

\author{
Stoencheva $\mathrm{S}^{1 *}$, DenevaT ${ }^{1}$, Beleva $\mathrm{E}^{2}$, Grudeva Popova $\mathrm{Zh}^{2}$ and Popov $\mathrm{V}^{2}$ \\ ${ }^{1}$ Department of Clinical Laboratory, Bulgaria \\ ${ }^{2}$ Department of Clinical Oncology, Bulgaria \\ *Corresponding author: Stoencheva S, Department of Clinical Laboratory, Bulgaria
}

ARTICLE INFO

Received: 彗 June 26, 2019

Published: 豐 July 03, 2019

Citation: Stoencheva S, Deneva T, Beleva E, Grudeva Popova Zh, Popov V. Determination of Plasma Tissue Factor Antigen and Tissue Factor-Bearing Microparticles Activity in Healthy Subjects. Biomed J Sci \& Tech Res 19(3)2019. BJSTR. MS.ID.003292.

Keywords: Tissue Factor Antigen; Tissue Factor-Bearing Microparticles; Reference Values; Hemostasis; Hypercoagulation

\begin{abstract}
Background: Tissue factor (TF) is an integral membrane protein, normally separated from the blood by the vascular endothelium, which plays a key role in the initiation of blood coagulation. TF is present in plasma in various forms, including microparticles (MPs) and alternatively spliced TF. MPs are small $(<1 \mu \mathrm{m})$ membrane vesicles generated by activated or apoptotic cells. Levels of TF antigen (TF-Ag) and tissue factor-bearing microparticles (MP-TF) activity from different origins are thought to be associated with hypercoagulable states. The aim of the present study was to determine the reference range for plasma levels of TF-Ag and MP-TF activity in healthy subjects.
\end{abstract}

Methods: To establish the reference range for plasma levels of TF-Ag and MP-TF activity and study the impact of sex and age we recruited 120 healthy subjects of Bulgarian nationality aged between 18 and 65. The selection criteria for the reference group were made to comply with the generally approved recommendations of the International Federation of Clinical Chemistry (IFCC). Clinical and routine hematological, biochemical and coagulation tests were performed to assess their health status. Plasma levels of TFAg were determined using ELISA and MP-TF activity by means of bio-immunoassay.

Results: The reference range given as $95 \%$ of the measured values was defined for TF-Ag plasma levels with $59.2 \mathrm{pg} / \mathrm{ml}(90 \%$ CI: 50.9 -68.2) to $250.9 \mathrm{pg} / \mathrm{ml}$ ( $90 \%$ CI: 234.6$267.0)$ and for plasma levels of MP-TF activity with $0.03 \mathrm{pg} / \mathrm{ml}(90 \% \mathrm{CI}: 0.01-0.06)$ to $0.47 \mathrm{pg} / \mathrm{ml}$ (90\% CI: 0.43-0.50). We found no sex-related differences in plasma TF-Ag and MP-TF activity ( $\mathrm{p}>0.05$ ), which obviates the need for separate reference intervals for men and women. Single-factor dispersion analysis found no age dependency of plasma levels for TF-Ag and MP-TF activity ( $\mathrm{p}>0.05$ ) in the age range 18-65.

Conclusion: The reference values for TF-Ag and MP-TF activity plasma levels calculated according to the type of distribution of results can be used as baseline criteria in clinical laboratory studies and for clinical purposes.

\section{Introduction}

Tissue factor (TF) is a transmembrane glycoprotein that is essential for hemostasis. It binds the coagulation serine protease factor VII/VIIa (FVII/VIIa) to form a bimolecular complex that functions as the primary initiator of coagulation in vivo [1]. TF is expressed in the vascular adventitia, in astroglial cells, in organ capsules and is found in lungs and placenta at relatively high concentrations [2]. Unstimulated monocytes and endothelial cells do not express tissue factor but can be induced to express tissue factor on their surface under certain conditions, primarily by inflammatory cytokines and endotoxin [3-5]. Under normal circumstances cells in contact with blood do not express physiologically active TF. When mechanical or chemical damage of the vascular wall occurs, subendothelial TF is expressed/exposed to blood and binds plasma factor VIIa. The TF/factor VIIa complex initiates linked, enzymatic reactions that culminate in the generation of thrombin and the subsequent conversion of fibrinogen to fibrin 
[2]. Thrombin also activates platelets by cleavage of proteaseactivated receptors (PARs) [6]. TF is constitutively expressed at high levels in adventitial fibroblasts of the vessel wall and this facilitates the rapid activation of the coagulation cascade after injury $[7,8]$. Very low levels of TF are present in the blood of healthy individuals [9]. This so-called circulating TF is mostly present in the form of microparticles (MPs), which are submicron membrane vesicles $(<1 \mu \mathrm{m})$ derived from apoptotic and/or activated cells [10]. MPs have phospholipid surface which can assemble coagulation factors, resulting in procoagulant potential 2 . The role of circulating TF in healthy individuals has not been defined [11]. Blood also contains soluble forms of TF, including alternatively spliced TF [11].

Many studies have shown that levels of TF and MP-TF activity are increased in various diseases, including cancer, sepsis, cardiovascular diseases and diabetes [12-16]. Prothrombotic tendency is characteristic of solid tumors. Cancer is associated with a four-fold increase in the risk for venous thromboembolism (VTE) and chemotherapy further increases this risk to between six and seven-fold compared with that in the general population [17]. Overall, the occurrence of VTE in patients with cancer is associated with increased morbidity and mortality [18]. Tissue factor (TF) has been considered a crucial role in the cancer VTE, especially the expression of TF on microparticles (MPs) which were released from tumor cells in the circulating blood $[19,20]$.

TF expression has been reported in a number of cancers, such as prostate cancer, pancreatic cancer, ovarian cancer, non-smallcell lung cancer, colorectal cancer, renal cell cancer, hepatocellular cancer, glioma and breast cancer [21-24]. Treatment of patients with chemotherapeutic agents and/or radiation may additionally lead to an increase in the levels of MP-TF activity [19]. Monocytes are the major source of intravascular $\mathrm{TF}$ and are responsible for activation of coagulation during endotoxemia and sepsis [25]. Many studies have shown increased plasma levels of TF and MP-TF activity in cardiovascular diseases. Tissue factor plasma antigen is elevated in hypertensive patients and can be lowered by different classes of antihypertensive drugs [26]. It is observed that angiotensin II induces TF expression in monocytes, endothelial cells, and vascular smooth muscle cells [27]. On the other hand TF-MPs are released from atherosclerotic plaques, which leads to elevated TF plasma levels in patients with unstable angina and acute coronary syndromes [28]. It is also found that TF levels are elevated in diabetic patients13. The aim of the present study was to determine the reference range for plasma levels of TF-Ag and MPTF activity in contingent of Bulgarian people.

\section{Materials and Methods}

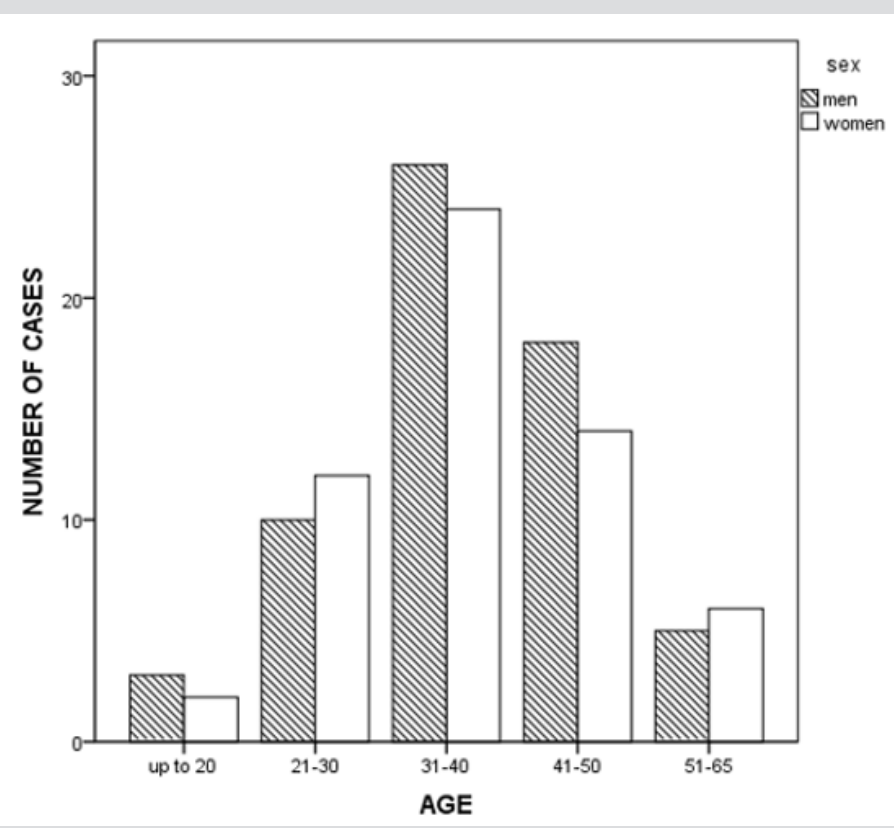

Figure 1: Distribution in the reference group by sex and age $(n=120)$.

We recruited 120 healthy subjects of Bulgarian nationality for a period of 1 year to establish the reference range for plasma levels of TF-Ag and MP-TF activity and study the impact of sex and age. The present clinical investigation was conducted according to the principles expressed in the Declaration of Helsinki. Ethical approval was obtained from the Scientific Ethics Committee of Medical University - Plovdiv. A written informed consent was obtained from all participants after explanation. The selection criteria for the reference group were made to comply with the generally approved recommendations of the International Federation of Clinical Chemistry (IFCC) [29]. The study included 62 men and 58 women, aged between 18 and 65: 109 were younger than 50 and 11 were older than 50 Figure 1 . Clinical and routine hematological, biochemical and coagulation tests were performed to assess their health status. Exclusion criteria were: diabetes mellitus or impaired glucose tolerance - fasting blood glucose $>6.1 \mathrm{mmol} / \mathrm{l}$; hypercholesterolemia; hypertriglyceridemia - TG > $1.7 \mathrm{mmol} / \mathrm{l}$; obesity - body mass index greater than $25 \mathrm{~kg} / \mathrm{m}^{2}$; cigarette smoking; 
clinical, instrumental (ECG, stress test) and laboratory evidence of coronary heart disease, hypertension, chronic obstructive pulmonary disease, bronchial asthma, peripheral vascular disease; chronic renal and liver diseases; systemic diseases of the connective tissues - lupus erythematodes, rheumatoid arthritis, collagenosis; acute inflammation or chronic inflammatory process in active treatment; neoplasma, prolonged use (during the last three months and during the time of study) of corticosteroids, hormonal drugs, psychotropic, narcotics; chronic use of alcohol and drug abuse. Plasma levels of TF antigen (TF-Ag) were analyzed by means of enzyme-linked immunosorbent assay (ELISA kit, Abcam, UK).

Levels of tissue factor - bearing microparticles (MP-TF) activity were analyzed by means of bio-immunoassay (Zymuphen MP-TF kit, HYPHEN Biomed, France). The major characteristics of analytic reliability of ELISA for TF-Ag were tested: intra-assay imprecision - CV $<3.7 \%$; inter-assay imprecision - $\mathrm{CV}<9.5 \%$; accuracy - d\% < 5.0\%; and recovery $89-114 \%$. For the bioimmunoassay of MP-TF activity were: intra-assay imprecision $\mathrm{CV}<6.8 \%$; inter-assay imprecision - CV < 9.2\%; accuracy - d\% < $4.8 \%$; and recovery $92-116 \%$. The methods show high precision; the results are consistent with the recommended minimal nonreproducibility (intra-assay imprecision - $\mathrm{CV}<3.9 \%$; inter-assay imprecision - CV $<9.9 \%$ for TF-Ag and intra-assay imprecision $\mathrm{CV}<8.0 \%$; inter-assay imprecision - CV $<10.0 \%$ for MP-TF activity respectively) for the methods as given by the manufacturers. The manners of withdrawal, processing and storage of blood samples and the choice of biological materials were in compliance with the requirements and the recommendations given by the manufacturer to compensate for the factors of result variation and for standardization of the preanalytical stage. Statistical calculations were carried out using IBM SPSS Statistics (v.25.0) and significance was fixed at $\mathrm{p}<0.05$. Kolmogorov-Smirnov test was used to assess normality distribution. The effect of sex was determined using the independent sample t-test. The reference intervals were calculated using IFCC algorithm developed for the software program REFVAL (v.3.21)29. The results were presented as central 95\% interval and 0.90 confidence interval of the reference range. Single-factor dispersion analysis was used to find if there is age dependency of plasma levels for TF-Ag and MP-TF activity.

\section{Results}

There were no statistically significant differences in the plasma levels of TF-Ag and MP-TF activity between males and females, which obviates the need for separate reference intervals for men and women Table 1. The distribution of the results for plasma levels of TF-Ag and MP-TF activity according to Kolmogorov - Smirnov test was Gaussian ( $p>0.05$ ) (Figures 2a \& 2b). The results of the statistical analysis of the data for plasma levels of TF-Ag and MP-TF activity are shown in Table 2. The reference limits with their $90 \%$ confidence interval were determined using parametric analysis. It is obvious that $\bar{x}$ and Me are similar, which is due to the regular distribution of the majority of the results. The reference range given as $95 \%$ of the measured values was defined for TF-Ag plasma levels with $59.2 \mathrm{pg} / \mathrm{ml}$ (90\% CI: 50.9-68.2) to $250.9 \mathrm{pg} / \mathrm{ml}$ (90\% CI: 234.6-267.0) and for plasma levels of MP-TF activity with $0.03 \mathrm{pg} /$ $\mathrm{ml}(90 \% \mathrm{CI}: 0.01-0.06)$ to $0.47 \mathrm{pg} / \mathrm{ml}$ (90\% CI: 0.43-0.50). Singlefactor dispersion analysis found no age dependency of plasma levels for TF-Ag and MP-TF activity ( $p>0.05)$, ( $F=1.226$; $F=0.650)$ in the age range of 18-65, which makes unnecessary establishment of reference intervals for smaller age range in this age group. We used graphic presentation to achieve easier interpretation of the interval structure of the reference interval (Figures 3a \& 3b).

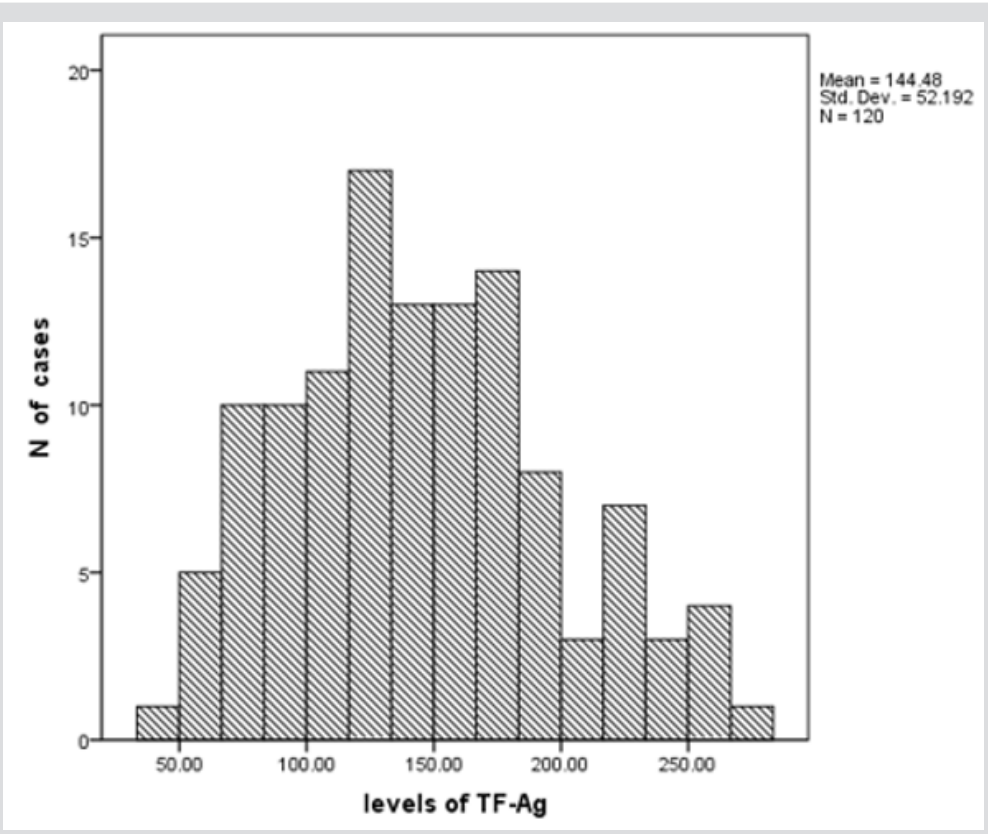

Figure 2a: Histogram of the results for TF-Ag plasma levels in the reference group. 


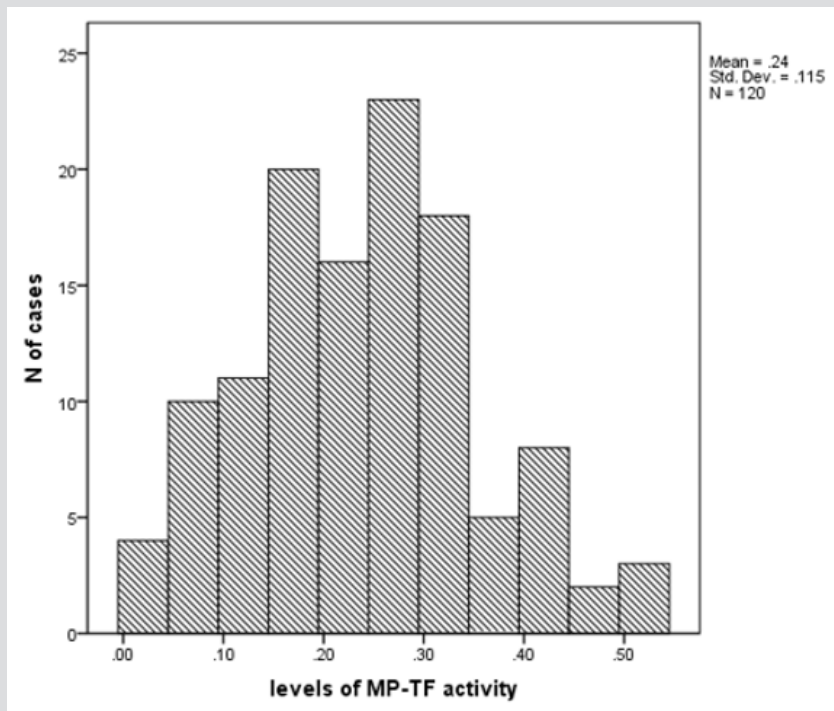

Figure 2b: Histogram of the results for MP-TF activity plasma levels in the reference group.

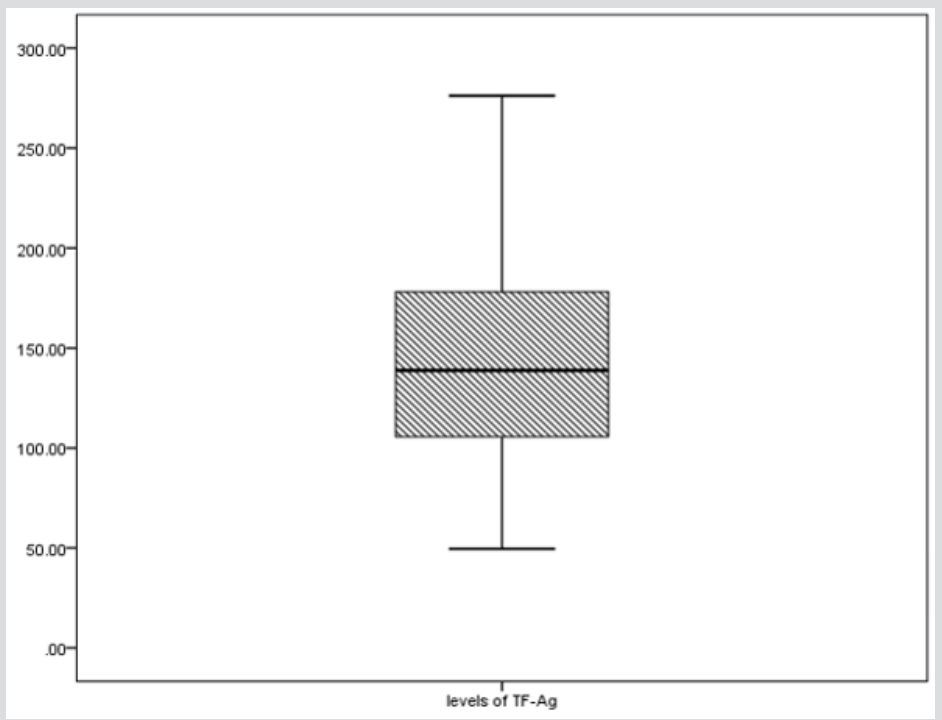

Figure 3a: Graphic presentation of the reference interval for plasma levels of TF-Ag in the reference group.

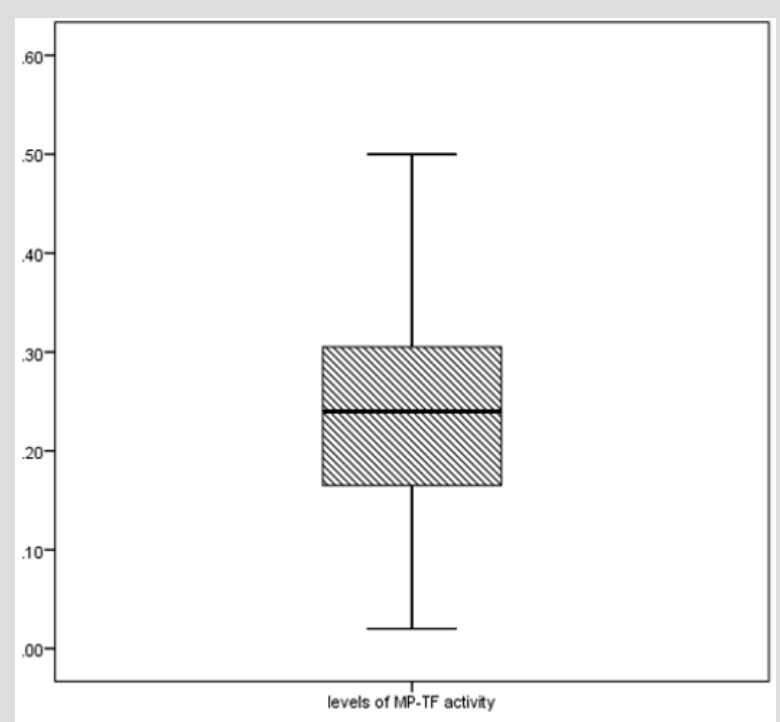

Figure 3b: Graphic presentation of the reference interval for plasma levels of MP-TF activity in the reference group. 
Table 1: Comparative characteristics of the results for TF-Ag and MP-TF activity plasma levels by sex.

\begin{tabular}{|c|c|c|c|c|c|c|}
\hline Parameters & sex & $\mathbf{N}$ & $\overline{\mathbf{x}}$ & SD & SEM & P \\
\hline levels of TF-MP & men & 62 & 0.2455 & 0.10967 & 0.01393 & 0.632 \\
\hline & women & 58 & 0.2353 & 0.12167 & 0.01598 & \\
\hline levels of TF-Ag & men & 62 & 147.1323 & 53.33095 & 6.77304 & 0.567 \\
\hline & women & 58 & 141.6466 & 51.25855 & 6.73058 & \\
\hline
\end{tabular}

$\overline{\mathrm{X}}$ - mean, SD - standard deviation, SEM - standard error of mean

Table 2: Reference intervals for TF-Ag and MP-TF activity.

\begin{tabular}{|c|c|c|}
\hline Parameters & TF-Ag pg/ml & MP-TF activity $\mathbf{~ p g} / \mathbf{m l}$ \\
\hline $\mathrm{N}$ & 120 & $0.02-0.54$ \\
\hline Range & $49.50-276.20$ & 0.24 \\
\hline Me & 138.8 & 0.24 \\
\hline $\bar{x}$ & 144.48 & 0.12 \\
\hline SD & 52.2 & 0.03 \\
\hline 0.025 fractile & 59.2 & $0.01-0.06$ \\
\hline $90 \%$ CI & $50.9-68.2$ & 0.47 \\
\hline 0.975 fractile & 250.9 & $0.43-0.50$ \\
\hline $90 \%$ CI & $234.6-267.0$ & \\
\hline
\end{tabular}

$\overline{\mathrm{x}}$-mean, SD - standard deviation, 90\% CI - 90\% confidence interval, Me- median

The reference range was plotted parallel to the Y-axis on which the units of measure of the parameter were marked. The horizontal lines mark the lower ( 0.025 fractile) and upper ( 0.975 fractile) of the reference ranges, the median ( 0.50 fractile) and the fractiles 0.25 and 0.75 . The two middle square margins form the central $50 \%$ fraction, while the two terminal margins include $22.5 \%$ of the results. The graphic presentation, which actually shows the internal structure of the result distribution, clearly indicates that the central $50 \%$ fraction presents practically symmetry distribution for TFAg and MP-TF activity plasma levels. This mode of presenting the reference interval has the advantage of providing immediate information about the type of distribution, the width and reliability of the reference intervals and the uniqueness of a specific result.

\section{Discussion}

\section{The Major Findings of The Present Study Are:}

a) The reference range given as $95 \%$ of the measured values was defined for TF-Ag plasma levels with $59.2 \mathrm{pg} / \mathrm{ml}(90 \%$ CI: $50.9-68.2)$ to $250.9 \mathrm{pg} / \mathrm{ml}$ (90\% CI: 234.6-267.0) and for plasma levels of MP-TF activity with $0.03 \mathrm{pg} / \mathrm{ml}$ (90\% CI: 0.01 0.06 ) to $0.47 \mathrm{pg} / \mathrm{ml}$ (90\% CI: $0.43-0.50)$.

b) We found no sex-related differences in the plasma levels of TF-Ag and MP-TF activity ( $p>0.05$ ), which obviates the need for separate reference intervals for men and women.

c) Single-factor dispersion analysis found no age dependency of levels for TF-Ag and MP-TF activity ( $p>0.05)$, ( $F=1.226$; F $=0.650$ ) in the age range of 18-65, which makes unnecessary establishment of reference intervals for smaller age ranges in this age group.
In spite of the fact that establishing reference values is an expensive and time - consuming task, it is recommended that they be determined in case of newly introduced parameters or parameters having genetic heterogeneity [29]. This is a reason for building reference intervals for a specific population from a specific region and with a specific structure. The present data of our study demonstrated that the mean level of TF-Ag is $144,48 \pm 52,18 \mathrm{pg} /$ $\mathrm{ml}$ (mean $\pm \mathrm{SD}$ ), which is consistent with the findings of other investigators. Plasma level of TF-Ag in healthy volunteers were within the range, observed by Quien et al. $(161 \pm 42 \mathrm{pg} / \mathrm{ml}$, mean \pm SD) and Takahashi et al. (138 $\pm 51 \mathrm{pg} / \mathrm{ml})[30,31]$. Our finding for the mean plasma level of MP - TF activity $0.24 \pm 0.12 \mathrm{pg} / \mathrm{ml}$ (mean $\pm \mathrm{SD}$ ) is in agreement with the findings of other studies, which have found that healthy individuals have very low levels of MP-TF activity [32,33]. Many studies have shown that plasma levels of TF$\mathrm{Ag}$ and MP-TF activity are increased in various diseases, including cardiovascular disease, sepsis, diabetes, and cancer12-16. The prevalence of VTE in cancer patients is approximately four-fold higher than in the general population17. This generally leads to a poor prognosis and is the second leading cause of death in cancer patients18. Increased plasma levels of TF-Ag and MP-TF activity are associated with hypercoagulable state in these patients, which requires the determination of reliable discriminant values (cut-off) to evaluate the risk of appearance of incidents of thrombosis.

\section{Conclusion}

The reference values for plasma levels of TF-Ag and MP-TF activity are calculated according to the type of result distribution; they can be used as baseline criteria for clinical laboratory purposes. The changes in the concentration of analyzed parameters for the age range 18-65 are not statistically significant, which means that it is 
not necessary to establish reference intervals for smaller age range within this group. The unavailability of sex-related differences in plasma levels of TF-Ag and MP-TF activity does not require separate reference intervals for men and women. The graphical model of reference interval provides immediate information about the type of distribution, the width and reliability of the reference values and makes it possible to easily visualize.

\section{Acknowledgement}

The study was supported by Medical University of Plovdiv, Project SDP № 05/2017.

\section{References}

1. Mackman N (2004) Role of tissue factor in hemostasis, thrombosis, and vascular development. Arterioscler Thromb Vasc Biol 24(6): 1015-1022.

2. Zwicker JI, Trenor CC, Furie BC, Furie B (2011) Tissue factor-bearing microparticles and thrombus formation. Atheroscler Thromb Vasc Biol 31(4): 728-733.

3. Bouchard BA, Tracy PB (2003) The participation of leukocytes in coagulant reactions. J Thromb Haemost 1(3): 464-469.

4. Nilziel M, van Oerle R, van't Veer C, Pampus E, Lindhout T, et al. (2001) Tissue factor activity in human monocytes is regulated by plasma: implications for the high and low responder phenomenon. Br J Haematol 112(1): 98-104.

5. Atkinson BT, Jasuja R, Chen V, Nandivada P, Furie B, et al. (2010) Laserinduced endothelial cell activation supports fibrin formation. Blood 116(22): 4675-4683.

6. Coughlin SR (2000) Thrombin signaling and protease-activated receptors. Nature 407(6801): 258-264.

7. Mackman N (1995) Regulation of the tissue factor gene. FASEB J 9(10): 883-889.

8. Osterud B, Bjorklid E (2006) Sources of tissue factor. Semin Thromb Hemost 32(1): 11-23.

9. Kasthuri RS, Taubman MB, Mackman N (2009) Role of Tissue Factor in Cancer. J Clin Oncol 27(29): 4834-4838.

10. Morel O, Toti F, Hugel B, Camoin-Jau L, Dignat-George F, et al. (2006) Procoagulant microparticles: Disrupting the vascular homeostasis equation? Arterioscler Thromb Vasc Biol 26(12): 2594-2604.

11. Giesen PL, Rauch U, Bohrmann B, Kling D, Roqué M, et al. (1999) Bloodborne tissue factor: Another view of thrombosis. Proc Natl Acad Sci U S A 96(5): 2311-2315.

12. Iacoviello L, Di Castelnuovo A, de Curtis A, Agnoli C, Frasca G et al. (2015) Circulating Tissue Factor Levels and Risk of Stroke: Findings from the EPICOR Study. Stroke 46(6): 1501-1507.

13. Soma P, Swanepoel AC, Bester J, Pretorius E (2017) Tissue factor levels in type 2 diabetes mellitus. Inflamm Res 66(5): 365-368.

14. Geddings JE, Mackman N (2013) Tumor-derived tissue factor-positive microparticles and venous thrombosis in cancer patients. Blood 122(11): 1873-1880.

15. Date K, Ettelaie C, Maraveyas A (2017) Tissue factor-bearing microparticles and inflammation: a potential mechanism for the development of venous thromboembolism in cancer. J Thromb Haemost 15(12): 2289-2299.

16. Tremoli E (2015) Tissue Factor in Arterial and Venous Thrombosis: From Pathophysiology to Clinical Implications. Semin Thromb Hemost 41(7): 680-681.
17. Falanga A, Russo L (2012) Epidemiology, risk and outcomes of venous thromboembolism in cancer. Hamostaseologie 32(2): 115-125.

18. Khorana AA, Francis CW, Culakova E, Kuderer NM, Lyman GH, et al. (2007) Thromboembolism is a leading cause of death in cancer patients receiving outpatient chemotherapy. J Thromb Haemost 5(3): 632-634.

19. Ruf W (2012) Tissue factor and cancer Thromb Res 130(suppl 1): S8487.

20. Manly DA, Wang J, Glover SL Kasthuri R, Liebman HA, et al. (2010) Increased microparticle tissue factor activity in cancer patients with venous thromboembolism. Thromb Res 125(6): 511-512.

21. Sartori MT, Della Puppa A, Ballin A, Campello E, Radu CM et al. (2013) Circulating microparticles of glial origin and tissue factor bearing in high-grade glioma: a potential prothrombotic role. Thromb Haemost 110(2): 378-385

22. Gomes FG, Sandim V, Almeida V (2017) Breast-cancer extracellular vesicles induce platelet activation and aggregation by tissue factorindependent and -dependent mechanisms. Thromb Res 159: 24-32.

23. Uno K, Homma S, Satoh T. Nakanishi K, Abe D, et al, (2007) Tissue factor expression as a possible determinant of thromboembolism in ovarian cancer. Br J Cancer 96(2): 290-295.

24. Regina S, Rollin J, Blechet C, Iochmann S, Reverdiau P, et al. (2008) Tissue factor expression in non-small cell lung cancer: relationship with vascular endothelial growth factor expression, microvascular density, and K-ras mutation. J Thorac Oncol 3(7): 689- 697.

25. Pawlinski R, Mackman N (2010) Cellular Sources of Tissue Factor in Endotoxemia and Sepsis. Thromb Res 125S1: S70-S73.

26. Felmeden DC, Spencer CG, Chung NA, Belgore FM, Blann AD, et al. (2003) Relation of thrombogenesis in systemic hypertension to angiogenesis and endothelial damage/dysfunction (a substudy of the AngloScandinavian Cardiac Outcomes Trial [ASCOT]). Am J Cardiol 92(4): 400-405.

27. He M, He X, Xie Q Chen F, He S, et al. (2006) Angiotensin II induces the expression of tissue factor and its mechanism in human monocytes. Thromb Res 117(5): 579-590.

28. Mallat Z, Benamer H, Hugel B, Benessiano J, Steg PG, et al. (2000) Elevated levels of shed membrane microparticles with procoagulant potential in the peripheral circulating blood of patients with acute coronary syndromes. Circulation 101(8): 841-843.

29. Solberg HE (1987) Approved recommendation of the theory of reference values. Part 5. Statistical treatment of collected reference values. Determination of reference limits. J Clin Chem Clin Biochem 25: 645-656.

30. Quien ET, Morales E, Cisar LA, Kim HC, Cimini C, et al. (1997) Plasma tissue factor antigen levels in capillary whole blood and venous blood: Effect of tissue factor on prothrombin time. Am J Hematol 55(4): 193198.

31. Takahashi H, Satoh N, Wada K, Takakuwa E, Seki Y, et al. (1994) Tissue factor in plasma of patients with disseminated intravascular coagulation. Am J Hematol 46(4): 333-337.

32. Khorana AA, Francis CW, Menzies KE, Wang JG, Hyrien O, et al. (2008) Plasma tissue factor may be predictive of venous thromboembolism in pancreatic cancer. J Thromb Haemost 6(11): 1983-1985.

33. Van Doormaal F, Kleinjan A, Berckmans RJ, Mackman N, Manly D, et al. (2012) Coagulation activation and microparticle-associated coagulant activity in cancer patients. An exploratory prospective study, Thromb. Haemost 108(1): 160-165. 


\section{ISSN: 2574-1241}

DOI: 10.26717/BJSTR.2019.19.003292

Stoencheva S. Biomed J Sci \& Tech Res

(c) (i) This work is licensed under Creative

Submission Link: https://biomedres.us/submit-manuscript.php

$\begin{array}{ll}\text { BIOMEDICAL } & \text { Assets of Publishing with us } \\ \text { RESEARCHES } & \text { - Global archiving of articles } \\ & \text { - Immediate, unrestricted online access } \\ & \text { - Rigorous Peer Review Process } \\ \end{array}$

